\title{
Um sistema baseado em PLN e AG para apoiar a mediação pedagógica em fóruns de discussão
}

Title: A system based on NLP and GA to support pedagogical mediation in discussion forums

\author{
Máverick Dionísio \\ Universidade Federal Rural de \\ Pernambuco \\ amaverick70@gmail.com
}

\author{
Vitor Belarmino Rolim \\ Universidade Federal Rural de \\ Pernambuco \\ vitor.rolim@ufrpe.br
}

\author{
Rafael Ferreira \\ Universidade Federal Rural de \\ Pernambuco \\ rafaelflmello@gmail.com
}

\author{
Anderson Pinheiro Cavalcanti \\ Universidade Federal Rural de \\ Pernambuco \\ anderson.pinheiro27@gmail.com
}

\author{
Cícero Garrozi \\ Universidade Federal \\ Rural de Pernambuco \\ cicero.garrozi@ufrpe.br
}

\section{Resumo}

Com o crescimento da Educação à Distância (EAD) os fóruns de discussão têm sido amplamente utilizados para promover interação e colaboração entre estudantes e professores de maneira assíncrona. Apesar dos beneficios para o processo de ensino e aprendizagem a utilização dos fóruns na EAD pode significar sobrecarga para os professores/tutores dada a grande quantidade de informações geradas nos debates. Levando em consideração a importância da atuação docente no acompanhamento das discussões e consequente auxílio aos estudantes da EAD, a sobrecarga do professor/tutor se enquadra como um problema. Diante disso, este estudo apresenta um sistema que integra Processamento de Linguagem Natural e Algoritmo Genético para classificar postagens de fóruns de discussão como Dúvida, Comentário Neutro ou Resposta. Como forma de avaliar o desempenho do sistema proposto foram conduzidos experimentos em três bases de dados, com um total de 1769 postagens, chegando o sistema a obter médias de F-measures de 0.981, 0.997 e 0.988 na classificação das postagens como Dúvida, Comentário Neutro e Resposta, respectivamente. Após os experimentos os resultados foram analisados por meio do teste estatístico z com um intervalo de confiança de 95\%. Os resultados obtidos evidenciam o potencial do sistema proposto para identificar gêneros de postagens, oriundas de fóruns de discussão, podendo o professor/tutor utilizálo como apoio para direcionar seus esforços, por exemplo, para estudantes com eventuais Dúvidas na discussão corrente.

Palavras-Chave: Fóruns de Discussão; Classificar Postagens; Atuação Docente.

\begin{abstract}
With the growth of e-learning discussion forums have been widely used to promote interaction and collaboration between students and teachers asynchronously. Despite the benefits to the teaching and learning process the use of the forums in the e-learning can mean overload for the teachers/tutors given the large amount of information generated in the debates. Taking into consideration the importance of the teaching performance in the follow-up of the discussions and consequent help to the students of the e-learning, the teacher/tutor overload is considered a problem. Therefore, this study presents a system that integrates Natural Language Processing and Genetic Algorithm to classify postings from discussion forums such as Doubt, Neutral comment or Answer. As a way of evaluating the performance of the proposed system, experiments were conducted in three databases, with a total of 1769 posts, arriving at the average F-measures of 0.981, 0.997 and 0.988 in the classification of posts as Doubt, Neutral comment and Answer, respectively. After the experiments the results were analyzed by means of the $z$ statistical test with a confidence interval of $95 \%$. The results show the potential of the proposed system to identify post genres, from discussion forums, and the teacher/tutor can use it as a support to direct their efforts, for example, to students with any doubts in the current discussion.
\end{abstract}

Keywords: Discussion Forums; Classify Postings; Teaching Performance.

Cite as: Dionísio, M., Ferreira, R., Garrozi, C., Rolim, V. \& Cavalcanti, A. (2018). A system based on NLP and GA to support pedagogical mediation in discussion forums (Um sistema baseado em PLN e AG para apoiar a mediação pedagógica em fóruns de discussão). Brazilian Journal of Computers in Education (Revista Brasileira de Informática na Educação - RBIE), 26(3), 61-75. DOI: 10.5753/RBIE.2018.26.03.61 


\section{Introdução}

A educação à distância (EAD) tem ganhado espaço ao longo dos anos por possibilitar a ampliação do processo de ensino e aprendizagem para além dos muros das instituições de ensino. A Associação Brasileira de Educação a Distância (ABED), por meio do censo 2015, evidencia o aumento do número de matrículas em cursos a distância ofertados por órgãos públicos, instituições públicas municipais e instituições privadas em $60 \%, 50 \%$ e 45,46\%, respectivamente [ABED 2015].

Nesse contexto, os Ambientes Virtuais de Aprendizagem (AVA) são plataformas utilizadas para a disponibilização de materiais de apoio pedagógico, ferramentas de avaliação e comunicação entre os atores envolvidos na EAD [Bartholo et al. 2009]. Dentre as ferramentas integradas aos AVA, destacam-se os fóruns de discussões educacionais por permitirem que os participantes de uma dada discussão contribuam com postagens assíncronas e aprendam sobre o assunto discutido via colaboração [Xia et al. 2013].

Além do ganho para o processo de ensino e aprendizagem, a utilização do fórum na EAD potencializa a criação de laços afetivos entre estudantes e professores, por meio das interações nos debates, sendo a afetividade um fator importante para a permanência do aprendiz em cursos a distância [Carvalho e Lima 2015]. Por outro lado, fazer uso do fórum pode significar sobrecarga para os professores/tutores devido ao grande número de postagens geradas nas discussões [Dringus e Ellis 2005; Scheuer e McLaren 2008].

Assim, essa sobrecarga se torna um problema dada a importância da atuação docente na EAD, principalmente na mediação dos fóruns, onde se espera auxílio aos estudantes no direcionamento das discussões, incentivo às interações do tipo estudante-estudante e resposta de dúvidas [Lim e Cheah 2010]. Vale ressaltar que ao pontuar a relevância dos feedbacks dos professores/tutores, para o andamento das discussões em fóruns, não é sugerido o mediador como detentor do conhecimento. Ao invés disso, entende-se o aprendiz da EAD como ativo, responsável por sua própria aprendizagem, ao mesmo tempo em que precisa de feedbacks para auxiliá-lo no processo de reflexão e acomodação dos conhecimentos adquiridos ao longo do debate [Abreu-e-Lima e Alves 2011].

Estudos recentes têm destacado a importância de integrar sistemas inteligentes em AVA com forma de apoiar o professor/tutor em atividades docentes na EAD. No contexto de fóruns educacionais, Nunes et al. (2014) apresentaram um método computacional, baseado em análise semântica e em técnicas estatísticas, direcionado para a identificação e exposição de tópicos relevantes em discussões assíncronas. Azevedo et al. (2011) disponibilizaram um software denominado mineraFórum designado à avaliação automática e qualitativa de postagens. Seguindo a mesma linha, Ferreira et al. (2016) experimentaram um modelo de avaliação qualitativa de postagens em um fórum educacional conduzido em língua portuguesa. É possível observar nos trabalhos citados um objetivo em comum, a utilização de métodos com potencial para automatizar processos e com isso desafogar o mediador em fóruns de discussão.

Apesar do potencial dos métodos apresentados, quando o objetivo é apoiar o professor/tutor no acompanhamento das discussões, esses apresentam limitações. Nesse aspecto, a classificação das postagens de fóruns como Dúvida, Comentário Neutro ou Resposta pode ajudar professores/tutores no direcionamento de seus esforços para acompanhar os alunos. De forma mais específica, esta categorização facilita a identificação de quais estudantes estão com dúvidas, na discussão corrente, oportunizando o provimento de feedback mais rápido e direcionado, evitando a desmotivação do aprendiz ou até, em caso de respostas, a inserção de questões provocativas visando o aprofundamento da discussão. 
Diante deste contexto, e do número ascendente de postagens em discussões online, este trabalho tem como principal objetivo apresentar um sistema computacional que integra técnicas de Processamento de Linguagem Natural (PLN) e Algoritmo Genético (AG) para classificar postagens de fóruns de discussão como Dúvida, Comentário Neutro ou Resposta.

Como forma de avaliar o desempenho do sistema proposto foram conduzidos experimentos em três bases de dados, com um total de 1769 postagens, utilizando as métricas precision, recall e $F$-measure além de avaliação com testes estatísticos. Como resultados o sistema obteve médias de F-measure de 0,981 (Dúvida), 0,997 (Comentário Neutro) e 0,988 (Resposta).

\section{Trabalhos Relacionados}

Esta seção lista trabalhos relacionados à identificação de gêneros de postagens de fóruns de discussão.

Qu e Liu (2012) propõem um etiquetador para postagens que considera a dependência entre as postagens como forma de inferir se um post retrata um problema (pergunta), uma solução para o problema (resposta), uma avaliação da resposta (confirmação) ou diversos. Com isso, utilizando o modelo conditional random field (CRF) o etiquetador proposto alcançou $F$ measure de 0,80 na classificação das postagens da classe pergunta.

Em Lin et al. (2009) é apresentado um sistema voltado para a identificação automática do gênero das postagens de fóruns conduzidos em língua chinesa. Os gêneros considerados foram: Anúncio, Pergunta, Esclarecimento, Interpretação, Conflito, Afirmação e Outros (tradução nossa). Os autores utilizaram uma base de dados com 9178 postagens e o classificador J48 o qual obteve até 0,717 de $F$-measure nos experimentos realizados.

No cenário brasileiro é possível identificar alguns trabalhos nesse sentido, em Guimarães e Esmin (2014) é realizada a classificação automática de postagens de fóruns educacionais nos seguintes gêneros: Anúncio, Dúvida, Esclarecimento, Interpretação e Outros. Para isso, foram extraídas e rotuladas 6367 postagens as quais foram utilizadas em um experimento com os classificadores Naive Bayes e Support Vector Machine (SVM) atingindo 0,608 e 0,603 de Fmeasure respectivamente.

Rolim et al. (2016) concentraram-se na identificação de dúvidas em fóruns educacionais e, como parte do processo, classificaram as postagens nas classes Dúvida, Neutra e Resposta. Para tal, conduziram experimentos em duas bases de dados com um total de 1090 postagens com os classificadores Naive Bayes, J48 e Multilayer Perceptron e como resultados alcançaram 0,97 de F-measure.

Apesar do resultado expressivo obtido por [Rolim et al. 2016], chama a atenção a utilização do número de interrogações como elemento no vetor de características das postagens classificadas. Contudo, na língua portuguesa, perguntas podem ser diretas ou indiretas (sem interrogação), por isso em escala maior esse trabalho pode apresentar problemas.

Os trabalhos anteriores utilizam palavras, pontuações, tamanho da postagem, entre outros, como características para identificação do gênero das postagens. Desse modo emerge a seguinte pergunta de pesquisa: Como identificar automaticamente o gênero de postagens em fóruns de discussões educacionais levando em consideração apenas as palavras? Esta questão é relevante, pois alguns caracteres podem direcionar o classificador para um resultado errado; por exemplo, a utilização de "?” para identificar dúvida pode não reconhecer perguntas indiretas. 
$\mathrm{Na}$ tentativa de responder a esta pergunta o presente trabalho se diferencia dos demais por apresentar um sistema computacional híbrido que integra Processamento de Linguagem Natural, Algoritmo Genético e o classificador Support Vector Machine (SVM) para identificar os gêneros (Dúvida, Neutra e Resposta) de postagens em Português.

\section{Fundamentação Teórica}

Para uma melhor compreensão dos aspectos que envolvem a presente pesquisa, nesta seção são explicitados os principais conceitos utilizados no trabalho.

\subsection{Algoritmo Genético}

Os AG são inspirados na teoria da evolução Darwiniana sendo amplamente aplicados no contexto de busca por soluções de problemas computacionais. Apesar de não garantir soluções ótimas, o AG é uma boa opção para reduzir o custo computacional ao mesmo tempo em que se obtêm soluções aproximadas [Farhan et al. 2015]. A propósito, dentre as diversas aplicações, os AG têm sido bastante utilizados para encontrar melhores conjuntos de parâmetros para algoritmos de aprendizagem de máquina [Grefenstette 1986] e em tarefas de agrupamento de textos [Afonso 2016].

O funcionamento de um AG simples [Melanie 1999] consiste na criação e evolução de uma população de soluções (indivíduos) para o problema em questão. Cada indivíduo é representado por um cromossomo, sendo este composto de genes binários ( $0 \mathrm{~s}$ e $1 \mathrm{~s})$, como exemplifica a Tabela 1.

Tabela 1: Exemplo de um cromossomo binário

\begin{tabular}{l|l|l|l|l|l|l|l}
\hline $\mathbf{1}$ & $\mathbf{1}$ & $\mathbf{0}$ & $\mathbf{0}$ & $\mathbf{0}$ & $\mathbf{1}$ & $\mathbf{0}$ & $\mathbf{1}$ \\
\hline
\end{tabular}

Com o objetivo de evoluir as soluções o AG simples funciona da seguinte maneira: (1) população inicial - gera aleatoriamente uma população de soluções (indivíduos) para o problema em questão; (2) Avaliação da população - verifica o quanto uma solução (indivíduo) se encontra adaptada ao problema; (3) seleção de pais - escolhe as soluções (indivíduos) mais adaptadas ao problema para participarem do cruzamento e/ou mutação; (4) cruzamento mistura do material genético das soluções selecionadas (indivíduos pais) com o objetivo de gerar novas soluções (indivíduos filhos) capazes de explorar novos espaços no campo de busca; (5) mutação - insere diversidade na população por meio da alteração (mutação) de alguns genes dada uma probabilidade definida a priori; (6) seleção de sobreviventes - com a geração de novas soluções (filhos) o número de indivíduos na população duplica e por isso é preciso selecionar os indivíduos que farão parte da próxima geração e; (7) atualização da população a partir da seleção de sobreviventes, atualiza a população com os indivíduos que farão parte da nova geração. As etapas listadas são executadas até atingirem um limite de execuções prédeterminado ou um critério de parada.

\subsection{Support Vector Machine (SVM)}

O SVM é um algoritmo de aprendizagem de máquina baseado na teoria do aprendizado estatístico [Vapnik 1995] e com diversas aplicações nos campos de classificação e categorização de textos [Joachims 2002]. Este algoritmo consegue lidar com problemas lineares e não-lineares. No caso de conjuntos de treinamento lineares são construídos hiperplanos a fim de conseguir separar, no plano cartesiano, os elementos a serem classificados. Um hiperplano é considerado como separação ótima se consegue separar os vetores das classes sem erro e com distância máxima para com os vetores mais próximos. 
Para conjuntos não-lineares são utilizadas as chamadas funções kernels, tais como: Polynomial, Rbf e Sigmoid. Estas permitem o mapeamento do conjunto de treinamento (nãolinear) para um espaço dimensional maior denominado de espaço de características. A partir disso, torna-se possível a separação linear.

\subsection{TF-IDF}

A medida estatística TF-IDF, segundo Salton e Yang (1973), é destinada à identificação do grau de importância de uma palavra para um conjunto de documentos. Assim, o seu cálculo consiste de três passos, como mostra as Equações (1, 2 e 3):

$$
\begin{gathered}
\mathrm{TF}=\frac{\text { número de vezes que o termo aparece em dado documento }}{\text { número total de termos presentes no documento }} \\
\mathrm{IDF}=1+\log \frac{\text { número total de documentos }}{\text { quantidade de documentos que apresentam determinado termo }}
\end{gathered}
$$

$$
\mathrm{TF}-\mathrm{IDF}=\mathrm{TF}^{*} \mathrm{IDF}
$$

Desse modo, o seu cálculo se resume em três passos, são eles: TF - frequência de uma palavra dentro do universo de documentos; IDF - número total de documentos existentes dividido pelo número total de documentos em que uma determinada palavra aparece e; TF-IDF a multiplicação do TF pelo IDF de cada palavra.

\subsection{Medidas de Avaliação}

$\mathrm{Na}$ área de aprendizagem de máquina medidas como recall, precision e $F$-measure (Equações 4 , 5 e 6) são bastante utilizadas para avaliar o desempenho de algoritmos de classificação [Ferri et al. 2009].

$$
\begin{gathered}
\text { precision }=\frac{\mathrm{VP}}{(\mathrm{VP}+\mathrm{FP})} \\
\text { recall }=\frac{\mathrm{VP}}{(\mathrm{VP}+\mathrm{FN})} \\
F \text {-measure }=\frac{2 * \text { precision } * \text { recall }}{\text { precision }+ \text { recall }}
\end{gathered}
$$

Onde, para calcular a precision e recall é utilizada como auxílio a chamada de matriz de confusão conhecida por tabelar os resultados obtidos da seguinte maneira: Verdadeiro positivo (VP) - número de elementos positivos classificados como positivos; Verdadeiro negativo (VN) - número de elementos positivos classificados como falsos; Falso positivo (FP) - número de elementos falsos classificados como positivos e; Falso negativo (FN) - número de elementos falsos classificados como falsos. Diante disso, Zhang e Zhang (2009) definem a F-measure como uma medida ponderada da precision e recall.

\subsection{Processamento de Linguagem Natural}

De acordo com Chowdhury (2003), a área da Computação que lida com informações fornecidas em língua natural, seja falada ou escrita, é denominada de Processamento de Linguagem Natural (PLN). Esta área busca entender como o ser humano entende e usa determinada língua como, por exemplo, o Português, a fim de projetar sistemas computacionais capazes de 
reconhecimento e síntese da fala humana, análise léxico-morfológica e entre outros [Vieira e Lima 2001].

Um exemplo de aplicações do PLN são os POS taggers (etiquetadores) capazes de analisar cada palavra ou termo contido em uma sentença de modo a atribuir ao item analisado, com base em sua função na sentença, uma classe gramatical [Vieira e Lima 2001]. Os etiquetadores podem ser projetados com base em regras, em modelos probabilísticos ou em regras e modelos probabilísticos (híbridos) [Santos et al. 2016]. As etiquetas atribuídas aos termos de uma sentença podem variar de acordo com o componente gramatical. O CoGrOO [Silva e Finger 2013] direcionado para a análise de textos, redigidos em português brasileiro, especificamente no que se refere à identificação de erros gramaticais, atribui aos termos analisados as etiquetas mostradas a seguir: n - substantivo; prop - nome próprio; art - artigo; pron - pronome; pron-pers - pronome pessoal; pron-det - pronome determinativo; pron-indp substantivo/pron-indp; adj - adjetivo; n-adj - substantivo/adjetivo; v - verbo; v-fin - verbo finitivo; v-inf - verbo infinitivo; v-pcp - verbo particípio; v-ger - verbo gerúndio; num numeral; prp - preposição; adj - adjetivo; conj - conjunção; conj-s - conjunção subordinativa; conj-c - conjunção coordenativa; intj - interjeição; adv - advérbio; xxx - outro.

\section{Sistema Proposto}

O sistema computacional proposto tem como objetivo classificar postagens de fóruns de discussão nas categorias Dúvida (D), Neutra (N) e Resposta (R). Para isso, foram utilizadas técnicas de PLN no processo de extração de características das postagens, o algoritmo SVM para classificação e o AG para encontrar o melhor conjunto de parâmetros para o SVM. O sistema proposto foi dividido em duas etapas: extração de características e classificação das postagens.

$\mathrm{Na}$ etapa de extração de características são retiradas das postagens as informações necessárias para o processo de classificação. Com isso, inicialmente é utilizado o componente gramatical CoGrOO [Silva e Finger 2013] para etiquetar todas as palavras das $n$ postagens de acordo com as suas respectivas funções gramaticais (POS) como exemplifica a Figura 1.

\begin{tabular}{|c|c|}
\hline \multicolumn{2}{|c|}{ Figura 1: Exemplo de etiquetagem } \\
\hline Postagens Dúvida (D) & Postagens Neutra (N) \\
\hline $\begin{array}{l}\text { o - pronome, que - pronome, } \\
\text { seria - verbo, um - artigo } \\
\text { sistema - substantivo, } \\
\text { integrado - verbo, ? - nenhum }\end{array}$ & $\begin{array}{l}\text { O - artigo, debate - substantivo, } \\
\text { está - verbo, legal - adjetivo, } \\
\text { ! - nenhum }\end{array}$ \\
\hline $\begin{array}{l}\text { O - pronome, que - pronome, } \\
\text { significa - verbo, } \\
\text { POO - substantivo, ? - nenhum }\end{array}$ & $\begin{array}{l}\text { Excelente - adjetivo, } \\
\text { discussão - substantivo, } \\
\text { ! - nenhum }\end{array}$ \\
\hline
\end{tabular}

Na sequência são criados os vetores de palavras das classes Dúvida, Neutra e Resposta considerando apenas as palavras com as seguintes funções gramaticais: Verbo, Substantivo, Pronome, Adjetivo e Advérbio. Nesse processo, as $n$ postagens são decompostas de modo a extrair todas as palavras e colocá-las dentro do vetor referente à sua classe de postagem (Dúvida, Neutra ou Resposta) e no subconjunto da função gramatical que desempenham (Verbo, Substantivo, Pronome, Adjetivo ou Advérbio) como demonstrado na Figura 2. 
Figura 2: Exemplo criação dos vetores de palavras

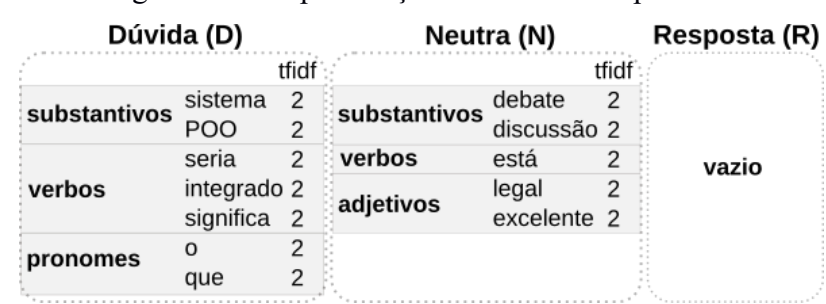

Após a distribuição das palavras nos vetores estas são ordenadas decrescentemente de modo a posicionar as palavras com maior representatividade, para cada classe gramatical, no início dos vetores. $\mathrm{O}$ processo de ordenamento considera uma medida de importância das palavras detalhada na seção 3.3. Por fim é gerado um vetor de características para cada postagem contendo 15 posições sendo considerada cada postagem de forma individual. Onde, cada posição do vetor recebe o número de palavras, com mesma função gramatical, que constam na postagem em questão e também em um dos vetores de palavras (Dúvida, Neutra e Resposta) (e.g., Figura 3).

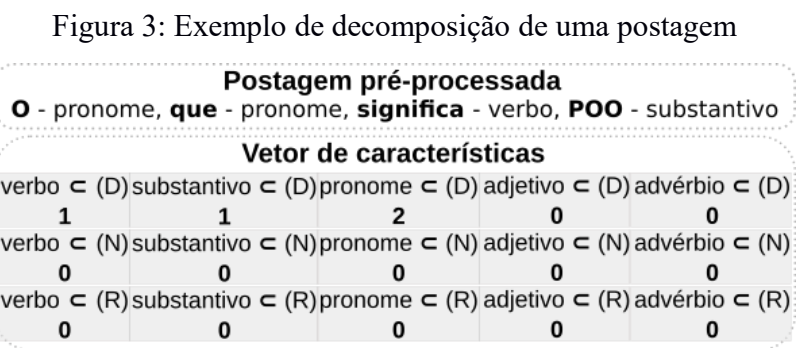

Por exemplo, a postagem utilizada na Figura 3 possui um verbo um substantivo e dois pronomes coincidentes com palavras de mesmas funções gramaticais presentes no vetor de palavras de Dúvidas. Após a preparação dos vetores de características, a classificação das postagens é realizada por meio do classificador SVM.

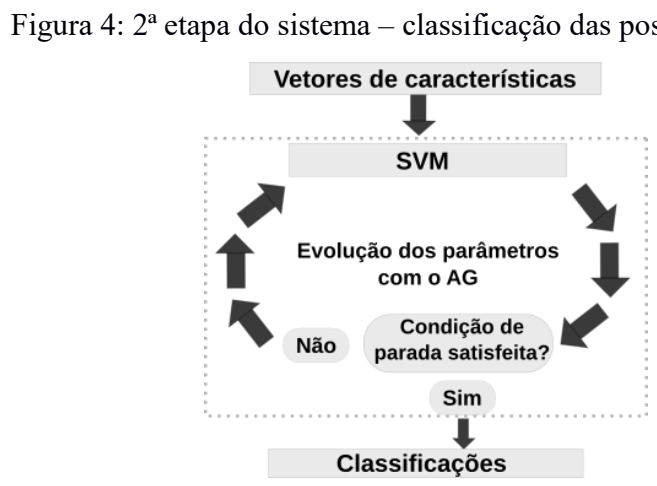

Como exibido na Figura 4, o SVM recebe os vetores de características e realiza o treinamento utilizando cada conjunto de parâmetros gerado pelo $A G$ até que uma condição de parada seja atingida. A propósito, o AG tem a sua população inicial representada por soluções (indivíduos) cujo material genético é constituído por oito genes que se referem aos parâmetros do SVM sintetizados na Tabela 2.

Tabela 2: Organização dos operadores de mutação para cada gene

\begin{tabular}{|c|c|c|c|c|c|c|c|}
\hline \multicolumn{2}{|c|}{ Mutação uniforme } & \multicolumn{5}{c|}{ Mutação gaussiana } \\
\hline svm_type & probability & kernel_type & Gamma & $N u$ & cache_size & Cost & Épsilon \\
\hline
\end{tabular}

O ciclo de execução do $A G$ é formado pelas seguintes fases: geração da população inicial - inicializa o algoritmo com indivíduos (soluções) compostos por parâmetros (genes) 
aleatórios; avaliação dos indivíduos - executa um SVM com o conjunto de parâmetros de cada indivíduo da população. Como o objetivo de cada indivíduo é parametrizar o SVM de modo a classificar corretamente as postagens, das classes Dúvida, Neutra e Resposta, a cada execução é calculada a F-measure que é utilizada como aptidão para cada indivíduo; avaliação dos indivíduos - Como o objetivo de cada indivíduo é parametrizar o SVM de modo a classificar corretamente as postagens, nas classes Dúvida, Neutra e Resposta, a avaliação de cada indivíduo é feita da seguinte maneira: executa um SVM, com o conjunto de parâmetros do respectivo indivíduo da população, fazendo uso da técnica cross-validation estratificada com 10 folds. Com isso, são obtidas três $F$-measures que correspondem ao desempenho do classificador para cada classe em questão. Por fim, é realizada a soma das F-measures e o resultado é dividido pelo número total de classes (no caso, três) resultando, assim, na aptidão final de cada indivíduo (conjunto de parâmetros); seleção de pais - escolhe indivíduos para participarem do cruzamento, sendo o método de seleção adotado a roleta por possibilitar aos indivíduos com baixa aptidão oportunidade de reprodução; cruzamento - mistura do material genético dos indivíduos selecionados (pais) com o objetivo de gerar novas soluções (filhos) capazes de explorar novos espaços no campo de busca, para tal é usado o cruzamento uniforme [Melanie 1999]; mutação - insere diversidade na população por meio da alteração (mutação) de alguns genes dada uma probabilidade definida a priori. Para isso, são utilizados dois operadores conforme exemplificado na Tabela 2: mutação uniforme - por possibilitar a escolha de dois genes aleatoriamente para posterior troca de posição e; mutação gaussiana - tendo em vista a ampla utilizando em problemas contínuos onde é gerado um valor a partir de uma distribuição normal $(0, \sigma)$, sendo $\sigma$ o desvio padrão 1 (um), para em seguida somar o valor gerado pela distribuição normal com o valor contido em um dos genes do indivíduo escolhido para mutação.

Seleção de sobreviventes - com a geração de novas soluções (filhos) o número de indivíduos na população duplica e por isso é preciso selecionar os indivíduos que farão parte da próxima geração. Nesse aspecto, utiliza-se o método steady state que consiste na seleção das melhores soluções dentro de um universo constituído pela população anterior e a população atual [Melanie 1999] e; atualização da população - atualiza a população de indivíduos, deixando as melhores soluções, com base na seleção realizada anteriormente.

\section{Avaliação do sistema}

Os procedimentos metodológicos utilizados para avaliar o sistema proposto seguem a estrutura apresentada na Figura 5.

Figura 5: Estrutura da metodologia

Seleção das
bases de dados $\begin{gathered}\text { Levantamento } \\ \text { de hipóteses }\end{gathered} \quad \begin{gathered}\text { Criação de } \\ \text { cenários }\end{gathered} \quad$ Experimentos Teste de hipótese

$\mathrm{Na}$ primeira etapa, seleção das bases de dados, três bases compostas por postagens oriundas de disciplinas da EAD foram selecionadas. Estas são referenciadas no decorrer do texto como BD1, BD2 e BD3.

As bases BD1 e BD2 são as mesmas utilizadas por Rolim et al. (2016) e foram extraídas do AVA da Universidade Federal de Alagoas (UFAL). O BD1 é composto por 490 postagens (198 - Dúvidas, 104 - Neutras e 188 - Respostas) de disciplinas do curso de bacharelado em Sistemas de Informação e o BD2 possui 600 postagens (200 - Dúvidas, 200 - Neutras e 200 Respostas) de disciplinas de Algoritmos e Estruturas de Dados. O BD3, também foi extraído do referido AVA, conta com um total de 679 postagens (60 - Dúvidas, 96 - Neutras e 523 - 
Respostas) obtidas das seguintes disciplinas: Análise de dados, Sistemas de Informação, Internet e Web e Programação Orientada a Objetos.

Ao levar em consideração as variáveis relacionadas ao classificador de gêneros proposto emergem algumas questões a considerar, são elas: considerar as funções gramaticais na atividade de montar os vetores de palavras (Dúvida, Neutra e Resposta) e os vetores de características das $n$ postagens melhora o desempenho do sistema? O uso do AG para ajustar os parâmetros do SVM implica em um melhor índice de classificação das postagens? O fato do sistema proposto não considerar caracteres como interrogações reduz o desempenho do sistema em comparação a trabalhos que utilizam?

Com base nos questionamentos listados, na etapa de levantamento de hipóteses, foram elaboradas 5 hipóteses, unicaudais à direita, pareadas (nula $\mathrm{H}_{0}$ e alternativa $\mathrm{H}_{\mathrm{a}}$ ) de tal forma que se uma for considerada falsa a outra será verdadeira (e.g., Tabela 3).

Tabela 3: Hipóteses levantadas

\begin{tabular}{|c|c|c|c|}
\hline & Hipótese nula & & Hipótese alternativa \\
\hline $\mathbf{H}_{\mathbf{0 a}}$ & $\begin{array}{c}\text { A F-measure obtida ao considerar as funções } \\
\text { gramaticais é menor ou igual à } F \text {-measure obtida sem } \\
\text { considerar as funções gramaticais. }\end{array}$ & $\mathrm{H}_{\mathrm{a} 1}$ & $\begin{array}{c}\text { A } F \text {-measure obtida ao considerar as funções } \\
\text { gramaticais é maior que a } F \text {-measure obtida sem } \\
\text { considerar as funções gramaticais. }\end{array}$ \\
\hline $\mathbf{H}_{0 b}$ & $\begin{array}{l}\text { A } F \text {-measure obtida com o uso do AG é menor ou } \\
\text { igual à } F \text {-measure obtida sem o uso do AG. }\end{array}$ & $\mathbf{H}_{\mathrm{a} 2}$ & $\begin{array}{l}\text { A } F \text {-measure obtida com o uso do AG é maior } \\
\text { que a } F \text {-measure obtida sem uso do AG. }\end{array}$ \\
\hline $\mathbf{H}_{0 \mathbf{c}}$ & $\begin{array}{c}\text { A F-measure obtida com o uso do AG e das funções } \\
\text { gramaticais é menor ou igual à } F \text {-measure obtida } \\
\text { apenas com o uso das funções gramaticais. }\end{array}$ & $\mathbf{H}_{\mathbf{a} 3}$ & $\begin{array}{l}\text { A } F \text {-measure obtida com o uso do AG e das } \\
\text { funções gramaticais é maior que a } F \text {-measure } \\
\text { obtida apenas com o uso das funções gramaticais. }\end{array}$ \\
\hline $\mathbf{H}_{0 \mathrm{~d}}$ & $\begin{array}{c}\text { A } F \text {-measure obtida com o uso do AG e das funções } \\
\text { gramaticais é menor ou igual à } F \text {-measure obtida } \\
\text { apenas com o uso do AG. }\end{array}$ & $\mathbf{H}_{\mathrm{a} 4}$ & $\begin{array}{c}\text { A } F \text {-measure obtida com o uso AG e das funções } \\
\text { gramaticais é maior que a } F \text {-measure obtida } \\
\text { apenas com o uso do AG. }\end{array}$ \\
\hline $\mathbf{H}_{\mathbf{0 e}}$ & $\begin{array}{l}\text { A } F \text {-measure obtida com o sistema apresentado neste } \\
\text { trabalho é menor ou igual à } F \text {-measure obtida com o } \\
\text { algoritmo proposto em Rolim et al. (2016). }\end{array}$ & $\mathbf{H}_{\mathrm{a} 5}$ & $\begin{array}{l}\text { A } F \text {-measure obtida com o sistema apresentado } \\
\text { neste trabalho é maior que a } F \text {-measure obtida } \\
\text { com o algoritmo proposto em Rolim et al. }(2016) \text {. }\end{array}$ \\
\hline
\end{tabular}

Como forma de verificar as hipóteses elencadas na Tabela 3, na etapa de criação de cenários, foram elaborados alguns cenários para classificação das postagens de cada base de dados: Cenário 1 (C1) - classificação das postagens por meio do sistema proposto; Cenário 2 (C2) - classificação das postagens por meio do sistema proposto sem considerar as funções gramaticais na criação dos vetores de palavras e dos vetores de características. Posteriormente, na etapa de experimentos, cada cenário foi executado 30 vezes com o módulo do AG ativado e 30 vezes com o módulo do AG desativado em cada configuração de corte (corte 0 , corte 5 e corte 10) dos $\mathrm{BD} 1, \mathrm{BD} 2$ e BD3, totalizando 540 execuções para cada cenário (dois cenários).

O tamanho do corte se refere a quanto em percentual se utiliza das palavras contidas nos vetores das classes Dúvida, Neutra e Resposta para montar os vetores de características. Por exemplo: corte 0 - utiliza $100 \%$ das palavras contidas nos vetores de palavras; corte 5 - utiliza 95\% das palavras contidas nos vetores de palavras e; corte 10 - utiliza $90 \%$ das palavras contidas nos vetores de palavras. As execuções que utilizaram o AG fizeram uso dos seguintes ajustes: 100 gerações, 30 indivíduos por geração, taxa de cruzamento de $50 \%$ e uma probabilidade de mutação de $2 \%$ para cada gene.

Os resultados alcançados pelo sistema proposto foram comparados com os de Rolim et al. (2016), até então, melhor resultado na literatura. O algoritmo supracitado foi executado, com sua melhor configuração, 30 vezes em cada configuração de corte (corte 0 , corte 5 e corte 10) dos BD1, BD2 e BD3 totalizando 270 execuções.

Para satisfazer a cada teste de hipótese, foram criadas 8 amostras a partir das execuções dos cenários 1 e 2, são elas: amostra de execuções com o módulo do AG ativado (540 
execuções); amostra de execuções com o módulo do AG desativado (540 execuções); amostra de execuções considerando as funções gramaticais (540 execuções); amostra de execuções sem utilizar as funções gramaticais (540 execuções); amostra de execuções considerando as funções gramaticais e com o módulo do AG ativado (270 execuções); amostra de execuções considerando as funções gramaticais e com o módulo do AG desativado (270 execuções); amostra de execuções sem utilizar as funções gramaticais e com o módulo do AG ativado (270 execuções) e; amostra de execuções do algoritmo de Rolim et al. (2016) (270 execuções).

Por fim, com o objetivo de escolher qual o teste estatístico utilizar, foi aplicado o teste de aderência Shapiro-Wilk obtendo-se p-values inferiores a 0.01 . Por isso, constatou-se com $99 \%$ de confiança que as amostras listadas acima não seguem uma distribuição normal. Somando a isto, as amostras são independentes e formadas a partir de ciclos de 30 execuções sem o uso de nenhum de tipo heurística para geração ou seleção dos resultados. Assim, para testar as hipóteses levantadas, foi adotado o teste não paramétrico de sinais de wilcoxon [Larson 2010].

\section{Experimentos}

Nesta seção são apresentados os resultados obtidos a partir dos experimentos nos BD1, BD2 e BD3. As tabelas exibidas a seguir apresentam em negrito as médias das $F$-mesuares obtidas (a cada ciclo de 30 execuções) e seus respectivos desvios padrões.

\subsection{Resultados do Cenário 1}

Os resultados obtidos com classificação das postagens por meio do sistema proposto $(\mathrm{C} 1)$ são exibidos na Tabela 4. Nesse Cenário, sem fazer uso do AG o sistema chegou a atingir no BD1 F-measure média de 0,946 (corte 0), 0,961 (corte 0) e 0,953 (corte 0) nas classes Dúvida, Neutra e Resposta. Para as mesmas classes o BD2 atingiu F-measures de 0,937 (corte 0), 0,943 (corte) e 0,979 (corte 0 ).

Tabela 4: Resultados do cenário 1

\begin{tabular}{|c|c|c|c|c|c|c|c|c|c|c|}
\hline & \multicolumn{3}{|c|}{ Corte 0} & \multicolumn{3}{|c|}{ Corte 5} & \multicolumn{3}{|c|}{ Corte 10} \\
\hline & & Dúvida & Neutra & Resposta & Dúvida & Neutra & Resposta & Dúvida & Neutra & Resposta \\
\hline \multirow{3}{*}{$\begin{array}{l}0 \\
0 \\
0 \\
\text { ¿ } \\
\text { ¿ }\end{array}$} & BD1 & $\begin{array}{c}\mathbf{0 , 9 4 6} \\
(0,0041)\end{array}$ & $\begin{array}{c}\mathbf{0 , 9 6 1} \\
(0,0050)\end{array}$ & $\begin{array}{c}\mathbf{0 , 9 5 3} \\
(0,0035)\end{array}$ & $\begin{array}{c}\mathbf{0 , 9 1 3} \\
(0,0033)\end{array}$ & $\begin{array}{c}\mathbf{0 , 9 2 7} \\
(0,0047)\end{array}$ & $\begin{array}{c}\mathbf{0 , 9 1 6} \\
(0,0020)\end{array}$ & $\begin{array}{c}\mathbf{0 , 9 1 7} \\
(0,0032)\end{array}$ & $\begin{array}{c}\mathbf{0 , 9 2 2} \\
(0,0048)\end{array}$ & $\begin{array}{l}17 \\
24)\end{array}$ \\
\hline & D2 & $\begin{array}{c}\mathbf{0 , 9 3 7} \\
(0,0041)\end{array}$ & $\begin{array}{c}\mathbf{0 , 9 4 3} \\
(0,0032)\end{array}$ & $\begin{array}{c}\mathbf{0 , 9 7 9} \\
(0,0018)\end{array}$ & $\begin{array}{c}\mathbf{0 , 1 6 0} \\
(0,0107)\end{array}$ & $\begin{array}{c}\mathbf{0 , 5 2 1} \\
(0,0021)\end{array}$ & $\begin{array}{r}\mathbf{0}, \\
(0,0\end{array}$ & & $\begin{array}{r}\mathbf{0}, \\
(0,0\end{array}$ & $\begin{array}{r}\mathbf{0} \\
(0,\end{array}$ \\
\hline & $\left.\right|^{\mathbf{D}}$ & $\begin{array}{c}\mathbf{0 , 8 7 4} \\
(0,008)\end{array}$ & $\begin{array}{c}\mathbf{0 , 9 7 9} \\
(0,0014)\end{array}$ & $\begin{array}{r}\mathbf{0 , 9} \\
(0,0\end{array}$ & $\begin{array}{c}\mathbf{0 , 8 2 6} \\
(0,0073)\end{array}$ & $\begin{array}{r}\mathbf{0}, \\
(0,0\end{array}$ & $\begin{array}{r}\mathbf{0}, \\
(0,0\end{array}$ & $\begin{array}{r}\mathbf{0 ,} \\
(0,0\end{array}$ & $\begin{array}{r}\mathbf{0}, \\
(0,,\end{array}$ & $\begin{array}{r}\mathbf{0} \\
(0,\end{array}$ \\
\hline \multirow{3}{*}{$\begin{array}{l}0 \\
0 \\
\Xi \\
0 \\
0\end{array}$} & B & $\begin{array}{c}\mathbf{0 , 9 7 8} \\
(0,0021)\end{array}$ & $\begin{array}{c}\mathbf{0 , 9 7 6} \\
(0,0024)\end{array}$ & $\begin{array}{r}\mathbf{0 , 9} \\
(0,0\end{array}$ & $\begin{array}{r}\mathbf{0 , 9} \\
(0,0\end{array}$ & $\begin{array}{r}\mathbf{0}, \\
(0,0\end{array}$ & $\begin{array}{r}\mathbf{0 ,} \\
(0, \mathrm{C}\end{array}$ & $\begin{array}{r}\mathbf{0 ,} \\
(0,0\end{array}$ & $\begin{array}{r}\mathbf{0}, \\
(0, \mathrm{C}\end{array}$ & $\begin{array}{r}\mathbf{0}, \\
(0,\end{array}$ \\
\hline & BD2 & $\begin{array}{c}\mathbf{0 , 9 8 1} \\
(0,003)\end{array}$ & $\begin{array}{r}\mathbf{0 , 9} \\
(0,0\end{array}$ & $\begin{array}{r}\mathbf{0 , 9} \\
(0,0\end{array}$ & $\begin{array}{r}\mathbf{0 , 9} \\
(0,0\end{array}$ & $\begin{array}{l}\mathbf{0}, \\
(0,\end{array}$ & $\begin{array}{r}\mathbf{0}, \\
(0,\end{array}$ & $\begin{array}{r}\mathbf{0}, \\
(0,\end{array}$ & $\begin{array}{r}\mathbf{0}, \\
(0,\end{array}$ & $\begin{array}{r}\mathbf{0}, \\
(0,\end{array}$ \\
\hline & BD3 & $\begin{array}{c}\mathbf{0 , 9 7 8} \\
(0,005)\end{array}$ & $\begin{array}{c}\mathbf{0 , 9 9 7} \\
(0,0006)\end{array}$ & $\begin{array}{c}\mathbf{0 , 9 8 8} \\
(0,003)\end{array}$ & $\begin{array}{c}\mathbf{0 , 9 5 7} \\
(0,002)\end{array}$ & $\begin{array}{c}\mathbf{0 , 9 6 4} \\
(0,0019)\end{array}$ & $\begin{array}{c}\mathbf{0 , 9 9 2} \\
(0,0004)\end{array}$ & $\begin{array}{c}\mathbf{0 , 9 3 0} \\
(0,002)\end{array}$ & $\begin{array}{c}\mathbf{0 , 9 6 4} \\
(0,002)\end{array}$ & $\begin{array}{c}\mathbf{0 , 9 8 9} \\
(0,0005)\end{array}$ \\
\hline
\end{tabular}

Seguido pelo o BD3 que, em todos os cortes, obteve resultados superiores a 0,90 . Utilizando o AG para evoluir os parâmetros do SVM os resultados foram potencializados em todos os cortes, com o AG, mostrando que no Cenário 1 o sistema não se restringiu a obter bons resultados da forma isolada (outliers) ao contrário disso manteve-se consistente em todas as bases de dados. 


\subsection{Resultados do Cenário 2}

O Cenário 2 sem a utilização do AG (e.g., Tabela 5) obteve as melhores médias de F-measure quando o corte estava em 0 , sendo interessante destacar alguns resultados, tais como: a classe Dúvida no BD1 com 0,870 (corte 0), a classe Neutra nos BD1 e BD2 com respectivas taxas de 0,98 (corte 0) e 0,99 (corte 0) e a classe Resposta no BD3 com 0,952 (corte 0).

Tabela 5: Resultados do cenário 1

\begin{tabular}{|c|c|c|c|c|c|c|c|c|c|c|}
\hline & \multicolumn{3}{|c|}{ Corte 0} & \multicolumn{3}{|c|}{ Corte 5} & \multicolumn{3}{|c|}{ Corte 10} \\
\hline & & Dúvida & Neutra & Resposta & Dúvida & Neutra & Resposta & Dúvida & Neutra & Resposta \\
\hline \multirow{3}{*}{ 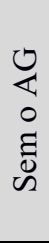 } & BD1 & $\begin{array}{l}\mathbf{0 , 8 7 0} \\
(0,00)\end{array}$ & $\begin{array}{c}\mathbf{0 , 9 8} \\
(0,0005)\end{array}$ & $\begin{array}{c}\mathbf{0 , 8 8} \\
(0,0003)\end{array}$ & $\begin{array}{c}\mathbf{0 , 1 3 7} \\
(0,0153)\end{array}$ & $\begin{array}{c}\mathbf{0 , 3 7 3} \\
(0,0012)\end{array}$ & $\begin{array}{c}\mathbf{0 , 1 7 3} \\
(0,0071)\end{array}$ & $\begin{array}{c}\mathbf{0 , 1 4 6} \\
(0,016) \\
\end{array}$ & $\begin{array}{c}\mathbf{0 , 3 7 3} \\
(0,0056)\end{array}$ & $\begin{array}{c}\mathbf{0 , 1 7 1} \\
(0,0022)\end{array}$ \\
\hline & BD2 & $\begin{array}{c}\mathbf{0 , 2 8 5} \\
(0,2296)\end{array}$ & $\begin{array}{c}\mathbf{0 , 6 2 1} \\
(0,1223)\end{array}$ & $\begin{array}{c}\mathbf{0 , 2 2 6} \\
(0,2471)\end{array}$ & $\begin{array}{c}\mathbf{0 , 4 2 4} \\
(0,2147)\end{array}$ & $\begin{array}{c}\mathbf{0 , 7 3 8} \\
(0,1441)\end{array}$ & $\begin{array}{c}\mathbf{0 , 4 9 7} \\
(0,3285)\end{array}$ & $\begin{array}{c}\mathbf{0 , 3 7 5} \\
(0,2223)\end{array}$ & $\begin{array}{c}\mathbf{0 , 7 0 5} \\
(0,1390) \\
\end{array}$ & $\begin{array}{c}\mathbf{0 , 4 3 2 9} \\
(0,3146)\end{array}$ \\
\hline & BD3 & $\begin{array}{c}\mathbf{0 , 2 8 3} \\
(0,0044)\end{array}$ & $\begin{array}{c}\mathbf{0 , 9 9 0} \\
(0,0029)\end{array}$ & $\begin{array}{c}\mathbf{0 , 9 5 2} \\
(0,0005)\end{array}$ & $\begin{array}{c}\mathbf{0 , 2 2 5} \\
(0,0182)\end{array}$ & $\begin{array}{c}\mathbf{0 , 9 6 3} \\
(0,0003)\end{array}$ & $\begin{array}{c}\mathbf{0 , 9 4 7} \\
(0,0010)\end{array}$ & $\begin{array}{c}\mathbf{0 , 2 5 9} \\
(0,0337)\end{array}$ & $\begin{array}{l}\mathbf{0 , 9 4 7} \\
(0,00)\end{array}$ & $\begin{array}{c}\mathbf{0 , 9 4 7} \\
(0,0010)\end{array}$ \\
\hline \multirow{3}{*}{ 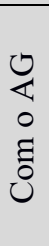 } & BD1 & $\begin{array}{c}\mathbf{0 , 8 9 6} \\
(0,0014)\end{array}$ & $\begin{array}{c}\mathbf{0 , 9 9 5} \\
(0,0)\end{array}$ & $\begin{array}{c}\mathbf{0 , 9 1 1} \\
(0,0011)\end{array}$ & $\begin{array}{c}\mathbf{0 , 8 3 8} \\
(0,026)\end{array}$ & $\begin{array}{c}\mathbf{0 , 9 5 0} \\
(0,0099)\end{array}$ & $\begin{array}{c}\mathbf{0 , 8 4 9} \\
(0,047)\end{array}$ & $\begin{array}{c}\mathbf{0 , 8 4 4} \\
(0,022)\end{array}$ & $\begin{array}{c}\mathbf{0 , 9 4 2} \\
(0,00221)\end{array}$ & $\begin{array}{c}\mathbf{0 , 8 7 6} \\
(0,012)\end{array}$ \\
\hline & BD2 & $\begin{array}{c}\mathbf{0 , 7 5 0} \\
(0,0401) \\
\end{array}$ & $\begin{array}{c}\mathbf{0 , 9 4 9} \\
(0,0114)\end{array}$ & $\begin{array}{c}\mathbf{0 , 8 3 4} \\
(0,0179) \\
\end{array}$ & $\begin{array}{c}\mathbf{0 , 7 2 1} \\
(0,0394)\end{array}$ & $\begin{array}{c}\mathbf{0 , 9 0 5} \\
(0,024) \\
\end{array}$ & $\begin{array}{c}\mathbf{0 , 8 2 5} \\
(0,825)\end{array}$ & $\begin{array}{c}\mathbf{0 , 6 9 7} \\
(0,019)\end{array}$ & $\begin{array}{c}\mathbf{0 , 8 7 7} \\
(0,018) \\
\end{array}$ & $\begin{array}{c}\mathbf{0 , 7 8 8} \\
(0,023)\end{array}$ \\
\hline & BD3 & $\begin{array}{c}\mathbf{0 , 5 4 9} \\
(0,0064)\end{array}$ & $\begin{array}{c}\mathbf{0 , 9 9 4} \\
(0,0)\end{array}$ & $\begin{array}{c}\mathbf{0 , 9 6 4} \\
(0,0002)\end{array}$ & $\begin{array}{c}\mathbf{0 , 4 7 6} \\
(0,0180)\end{array}$ & $\begin{array}{c}\mathbf{0 , 9 6 0} \\
(0,0004)\end{array}$ & $\begin{array}{c}\mathbf{0 , 9 7 3} \\
(0,0014)\end{array}$ & $\begin{array}{c}\mathbf{0 , 4 9 8} \\
(0,016)\end{array}$ & $\begin{array}{c}\mathbf{0 , 9 5 4} \\
(0,003)\end{array}$ & $\begin{array}{c}\mathbf{0 , 9 5 9} \\
(0,0007)\end{array}$ \\
\hline
\end{tabular}

Ao analisar a Tabela 5, de modo geral, conclui-se que os melhores resultados no Cenário 2 se deram nas execuções com o AG chegando às classes Dúvida, Neutra e Resposta a atingir médias de F-measure nessa ordem: 0,896 (corte 0), 0,995 (corte 0) e 0,973 (corte 5).

\subsection{Resultados do Algoritmo de Rolim et al.}

O algoritmo proposto por Rolim et al. (2016) foi executado em sua melhor configuração. Ao analisar os resultados (e.g., Tabela 6) observa-se que as melhores médias de F-measure aconteceram no BD1 e BD2.

Tabela 6: Resultado do algoritmo de Rolim et al. (2016)

\begin{tabular}{|c|c|c|c|c|c|c|c|c|c|c|}
\hline & \multicolumn{3}{|c|}{ Corte 0} & \multicolumn{3}{|c|}{ Corte 5} & \multicolumn{3}{|c|}{ Corte 10} \\
\hline & & Dúvida & Neutra & Resposta & Dúvida & \begin{tabular}{|l|} 
Neutra \\
\end{tabular} & Resposta & \begin{tabular}{|l|} 
Dúvida \\
\end{tabular} & Neutra & Resposta \\
\hline \multirow{3}{*}{ 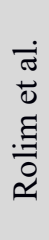 } & BD1 & \begin{tabular}{|c|}
$\mathbf{0 , 9 5 4}$ \\
$(0,0095)$
\end{tabular} & $\begin{array}{c}\mathbf{0 , 9 4 0} \\
(0,010)\end{array}$ & $\begin{array}{c}\mathbf{0 , 9 2 7} \\
(0,011)\end{array}$ & $\begin{array}{c}\mathbf{0 , 8 8 0} \\
(0,0071)\end{array}$ & \begin{tabular}{|c|}
$\mathbf{0 , 7 6 4}$ \\
$(0,014)$
\end{tabular} & $\begin{array}{c}\mathbf{0 , 8 3 7} \\
(0,009)\end{array}$ & $\begin{array}{c}\mathbf{0 , 8 4 7} \\
(0,0081)\end{array}$ & $\begin{array}{c}\mathbf{0 , 7 0 0} \\
(0,028)\end{array}$ & $\begin{array}{c}\mathbf{0 , 8 0 3} \\
(0,011)\end{array}$ \\
\hline & BD2 & $\begin{array}{c}\mathbf{0 , 9 6 3} \\
(0,0074)\end{array}$ & $\begin{array}{c}\mathbf{0 , 9 4 3} \\
(0,010)\end{array}$ & $\begin{array}{c}\mathbf{0 , 9 6 1} \\
(0,009)\end{array}$ & $\begin{array}{c}\mathbf{0 , 8 8 6} \\
(0,011)\end{array}$ & $\begin{array}{c}\mathbf{0 , 8 4 7} \\
(0,014)\end{array}$ & $\begin{array}{c}\mathbf{0 , 8 5 1} \\
(0,0011)\end{array}$ & $\begin{array}{c}\mathbf{0 , 8 7 5} \\
(0,009)\end{array}$ & $\begin{array}{c}\mathbf{0 , 8 0 3} \\
(0,009)\end{array}$ & $\begin{array}{c}\mathbf{0 , 8 2 9} \\
(0,010)\end{array}$ \\
\hline & BD3 & $\begin{array}{c}\mathbf{0 , 9 1 4} \\
(0,0189)\end{array}$ & $\begin{array}{c}\mathbf{0 , 9 1 4} \\
(0,020) \\
\end{array}$ & $\begin{array}{r}\mathbf{0 , 9 8 3} \\
(0,003) \\
\end{array}$ & $\begin{array}{c}\mathbf{0 , 8 1} \\
(0,009) \\
\end{array}$ & \begin{tabular}{|c|}
$\mathbf{0 , 6 5 4}$ \\
$(0,006)$ \\
\end{tabular} & $\begin{array}{c}\mathbf{0 , 9 4 1} \\
(0,006) \\
\end{array}$ & $\begin{array}{c}\mathbf{0 , 8 1 2} \\
(0,008) \\
\end{array}$ & \begin{tabular}{|c|}
$\mathbf{0 , 6 0 6}$ \\
$(0,026)$ \\
\end{tabular} & $\begin{array}{c}\mathbf{0 , 9 3 9} \\
(0,003) \\
\end{array}$ \\
\hline
\end{tabular}

Por outro lado, o algoritmo demonstrou dificuldade de classificação no BD3 especialmente nas classes Dúvida e Neutra. De acordo com Phua et al. (2004), algoritmos de aprendizagem de máquina são sensíveis a bases desbalanceadas, implicando em priorização das classes com maior número de exemplos. Por isso, entende-se que a distribuição dos dados do BD3 pode ter influenciado no desempenho do algoritmo de Rolim et al. (2016). Entretanto, é importante ressaltar que o sistema proposto neste estudo também classificou as postagens do BD3. Além disso, o maior número de postagens do tipo Respostas é comum se tratando de fóruns de discussão educacionais.

Outro fator que pode ter impactado no resultado é a existência de algumas postagens no BD3 onde os estudantes escrevem uma pergunta e a respondem na mesma postagem. Dessa forma, como o algoritmo de Rolim e colegas (2015) considera a frequência de interrogações para aferir se uma postagem trata-se de uma Dúvida, Comentário Neutro ou uma Resposta, postagens como as exemplificadas na Tabela 7 podem ter confundido o classificador. 
Tabela 7: Exemplos de postagens da classe Resposta no BD3

\begin{tabular}{c|c}
\hline Aluno 1 & $\begin{array}{r}\text { "O que é Java? É uma linguagem de programação orientada a objeto, atualmente pertence a } \\
\text { Oracle, mas foi desenvolvida pela Sun Microsystems na década de 90" }\end{array}$ \\
\hline Aluno 2 & $\begin{array}{c}\text { "O que é UML? UML é um acrônimo para a expressão Unified Modeling Language. Pela } \\
\text { definão de seu nome, vemos que UML é uma linguagem que define uma série de artefatos } \\
\text { que nosuda na tarefa de modelar e documentar os sistemas orientados a objetos que } \\
\text { desenvolvemos". }\end{array}$ \\
\hline
\end{tabular}

Os resultados mencionados acima não confrontam os divulgados em Rolim et al. (2016) apesar de serem inferiores aos divulgados pelos autores supracitados. Atribuem-se os resultados inferiores ao fato de ser sintetizado o desempenho médio do algoritmo com o número de execuções $\geq 30$.

\section{Testes de Hipóteses}

Os testes de hipóteses apresentados nesta seção foram realizados com um nível de significância de 0,05 (e.g., Tabela 8). Ao analisar os resultados obtidos, na Tabela 8 , nota-se que a hipótese nula $\mathrm{H}_{0 \mathrm{a}}$ foi rejeitada em todas as classes (Dúvida, Neutra e Resposta) devido aos valores de probabilidade inferiores ao nível de significância pré-estabelecido $(p<0,05)$.

\begin{tabular}{|c|c|c|}
\hline & & p-valor \\
\hline \multirow{3}{*}{$\mathbf{H}_{0 \mathbf{a}}$} & Dúvida & $2.2 \mathrm{e}-16$ \\
\hline & Neutra & \begin{tabular}{|c|c|}
0.00389 \\
\end{tabular} \\
\hline & Resposta & $2.2 \mathrm{e}-16$ \\
\hline \multirow{3}{*}{$\mathbf{H}_{0 \mathrm{~b}}$} & Dúvida & $2.2 \mathrm{e}-16$ \\
\hline & Neutra & $2.2 \mathrm{e}-16$ \\
\hline & Resposta & $2.2 \mathrm{e}-16$ \\
\hline \multirow{3}{*}{$\mathbf{H}_{0 \mathrm{c}}$} & Dúvida & $2.2 \mathrm{e}-16$ \\
\hline & Neutra & $2.2 \mathrm{e}-16$ \\
\hline & Resposta & $2.2 \mathrm{e}-16$ \\
\hline \multirow{3}{*}{$\mathbf{H}_{0 \mathrm{~d}}$} & Dúvida & $2.2 \mathrm{e}-16$ \\
\hline & Neutra & 0.03277 \\
\hline & Resposta & $2.2 \mathrm{e}-16$ \\
\hline
\end{tabular}

Portanto, a F-measure obtida ao considerar as funções gramaticais para criar os vetores de palavras e os vetores de características é superior à obtida quando não consideradas as funções gramaticais.

As probabilidades obtidas na hipótese $\mathrm{H}_{0 \mathrm{~b}}$ em todas as classes de postagem foram de 2.2e16 e por serem inferiores à probabilidade de 0,05 implicam na rejeição da hipótese nula com $95 \%$ de confiança. Com isso, a hipótese alternativa é aceita evidenciando os melhores resultados alcançados pelo sistema com o uso do AG em comparação aos resultados obtidos com o sistema sem a utilização do AG.

A terceira hipótese $\mathrm{H}_{0 \mathrm{c}}$ também foi rejeitada em todas as classes de postagens. Tal rejeição evidencia um melhor desempenho do sistema ao utilizar conjuntamente funções gramaticais e o $\mathrm{AG}$ ao invés de considerar somente as funções gramaticais.

A hipótese $\mathrm{H}_{0 \mathrm{~d}}$, por sua vez, atingiu probabilidades de 2.2e-16, 0.03277 e 2.2e-16 todas inferiores 0,05 . Com isso, a hipótese nula $\left(\mathrm{H}_{0 \mathrm{~d}}\right)$ foi rejeitada mostrando que as F-measures alcançadas ao considerar as funções gramaticais e o AG são superiores às $F$-measures obtidas apenas com o uso do AG. 
Com base nos testes estatísticos, considerar as funções gramaticais para criar os vetores de palavras e os vetores de características influência positivamente no desempenho do sistema. Atribui-se isto ao fato de algumas palavras poderem desempenhar diferentes funções gramaticais em uma sentença. Por exemplo, a palavra "mais" pode ser etiquetada como Substantivo, Pronome, Preposição, Advérbio ou Conjunção.

Também foi evidenciado o potencial do algoritmo genético (AG) para evoluir os parâmetros do SVM, bem como a potencialização do sistema com a atuação conjunta do AG e das funções gramaticais.

Por fim, os resultados do sistema apresentado neste estudo considerando as funções gramaticais e utilizando o AG foram comparados com os do algoritmo de Rolim et al. (2016) (Seção 6.3). Com p-valores de 2.2e-16, para todas as classes de postagens, a hipótese $\mathrm{H}_{0 \mathrm{e}}$ também foi rejeitada (e.g., Tabela 9).

Tabela 9: Resultados dos testes comparativos entre o algoritmo proposto neste estudo e a proposta de Rolim et al. (2016)

\begin{tabular}{c|c|c}
\hline \multicolumn{2}{|c}{} & $p$-valor \\
\hline \multirow{3}{*}{$H_{0 g}$} & Dúvida & 2.2e-16 \\
\cline { 2 - 3 } & Neutra & $\mathbf{2 . 2 e - 1 6}$ \\
\cline { 2 - 3 } & Resposta & $\mathbf{2 . 2 e - 1 6}$ \\
\hline
\end{tabular}

Diante disso, conclui-se com 95\% de confiança que o sistema apresentado nesta pesquisa apresentou um melhor desempenho em termos de $F$-measure, no processo de identificação de gêneros (Dúvida, Neutra e Resposta) de postagens de fóruns de discussão, levando em consideração as três bases de dados utilizadas.

\section{Considerações Finais}

Este trabalho apresenta um sistema computacional para auxiliar o acompanhamento educacional de fóruns de discussão a partir da identificação de gêneros (Dúvida, Neutra e Resposta) de postagens. Para isso, o sistema integra técnicas de Processamento de Linguagem Natural (PLN), Algoritmo Genético (AG) e o classificador Support Vector Machine (SVM).

O sistema chegou a atingir $F$-measure média de 0,997 mostrando o benefício de se levar em consideração as funções gramaticais das palavras, no processo de criação dos vetores de características, quando o objetivo é identificar o gênero (Dúvida, Neutra ou Resposta) de uma postagem de um fórum de discussão educacional. Os resultados evidenciam também o ganho em termos de desempenho do classificador SVM quando parametrizado utilizando o AG.

Como trabalhos futuros, projeta-se integrar ao sistema apresentado módulos de classificação de postagens que contribuam para a identificação de aspectos relacionados à interação do tipo estudante-estudante em fóruns de discussão, tais como: reconhecimento de presença social, acolhimento ou reflexão das perspectivas dos outros estudantes e compartilhamento de informações.

\section{Agradecimentos}

Os autores agradecem à Coordenação de Aperfeiçoamento de Pessoal de Nível Superior (CAPES) pelo financiamento desta pesquisa. 


\section{Referências}

ABED. (2015). Relatório analítico da aprendizagem a distância no Brasil. Retrieved from http://abed.org.br/arquivos/Censo EAD 2015 POR.pdf

Abreu-e-Lima, D. M., Alves, M. N. (2011). O feedback e sua importância no processo de tutoria a distância. In: Pro-Posições, Campinas, v. 22, n.2. [GS SEARCH]

Afonso, A. R. (2016). Brazilian Portuguese Text Clustering Based on Evolutionary Computing. In: IEEE Latin America Transactions, v.14, n.7. [GS SEARCH]

Azevedo, B. F. T, Behar, P. A., Reategui, E. B. (2011). Análise das mensagens de fóruns de discussão através de um software para mineração de textos. In: XXII Simpósio Brasileiro de Informática na Educação, p. 20-29. Disponível em: http://www.brie.org/pub/index.php/sbie/article/view/1572. [GS SEARCH]

Bartholo, V. F., Amaral, M. A., Cagnin, M. I. (2009). Uma Contribuição para a Adaptabilidade de Ambientes Virtuais de Aprendizagem para Dispositivos Móveis. In: Brazilian Journal of Computers in Education, v.17, n.2, p. 36-47. doi: 10.5753/rbie.2009.17.02.36. Disponível em: http://br-ie.org/pub/index.php/rbie/article/view/97. [GS SEARCH]

Carvalho, M. R., Lima, R. L. (2015). A importância da afetividade na EaD: uma perspectiva de Wallon. In: Revista EDAPECI, v.15, n.1, p. 192-205. [GS SEARCH]

Chowdhury, G. G. (2003). Natural Language Processing. In: Annual Review of Information Science and Techonology, v.37, p. 51-89. [GS SEARCH]

Dringus, L. P., Ellis, T. (2005). Using data mining as a strategy for assessing asynchronous discussion forums. In: Computers \& Education, v.45, n.1, p. 141-160. doi: 10.1016/j.compedu.2004.05.003. [GS SEARCH]

Farhan, A. S., Tareq, W. Z., Awad, F. H. (2015). Solving N Queen Problem using Genetic Algorithm. In: Internacional Journal of Computer Applications, v.122, n.12. [GS SEARCH]

Ferri, C., Hernández-Orallo, J., Modroiu, R. (2009). An experimental comparison of perfomance measures for classification. In: Pattern Recognition, p. 27-38. [GS SEARCH]

Ferreira, M. A. D., Araújo, D. C., Melo, R. F., Nascimento, A., Cavalcanti, A. P., Silva, A. (2016). Análise de Classificadores para Avaliação automática em Fóruns Educacionais. In: Encontro Nacional de Inteligência Artificial e Computacional, Recife. [GS SEARCH]

Grefenstette, J. J. (1986). Optimization of Control Parameters for Genetic Algorithms. In: IEEE Transactions on Systems, v.16, n. 1. doi: 10.1109/TSMC.1986.289288. [GS SEARCH]

Guimarães, F. R., Esmin, A. A. A. (2014). Identificação Automática de Gêneros das Mensagens em Fóruns de Discussões do AVA. In: Anais do XI Encontro Nacional de Inteligência Artificial e Computacional. [GS SEARCH]

Joachims, T. (2002). Learning to classify texts using support vector machines: methods, theory and algorithms. Kluwer Academic Publishers. [GS SEARCH]

Larson, Ron. (2010). Estatística aplicada. 4. ed. São Paulo: Pearson Prentice Hall. [GS $\underline{\mathrm{SEARCH}}]$

Lim, C. P., Cheah, P. T. (2010). The Role of the Tutor in Asynchronous Discussion Boards: A Case Study of a Pre-Service Teacher Course. In: Educational Media International, p. 33-48. [GS SEARCH] 
Lin, F.; Hsieh, L., Chuang, F. (2009). Discovering genres of online discussion threads via text mining. In: Computers \& Education, v.52, n.2, p. 481-4959. doi: 10.1016/j.compedu.2008.10.005. [GS SEARCH]

Melanie, M. (1999). An introduction to genetic algorithms. A Bradford Book The MIT Press. Cambridge, Massachusetts • London, England. Fifth printing. [GS SEARCH]

Nunes, B. P. et al. (2014). A Topic Extraction Process for Online Forums. In: IEEE Internacional on Advanced Learning techonologies, p. 541-543. doi: 10.1109/ICALT.2014.158. [GS SEARCH]

Phua, C., Alahakoon, D., Lee, V. (2004). Minority Report in Fraud Detection: Classification of Skewed Data. ACM SIGKDD Explorations. v.6, p.50-59. doi: 10.1145/1007730.1007738. [GS SEARCH]

Qu, Z., Liu, Y. (2012). Sentence Dependency Tagging in Online Question Answering Forums. In: Proceedings of the 50th Annual Meeting of the Association for Computacional Linguistics, p. 554-562. [GS SEARCH]

Rolim, V. V., Ferreira, R., Costa, E. (2016). Identificação Automática de Dúvidas em Fóruns Educacionais. In: XXVII Simpósio Brasileiro de Informática na Educação (SBIE 2016), p. 936-945. doi: 10.5753/cbie.sbie.2016.936. Disponível em: http://www.brie.org/pub/index.php/sbie/article/view/6779. [GS SEARCH]

Salton, G., Yang, C. S. (1973). On the specification of term values in automatic indexing. In: Journal of Documentation, v.29, n.4, p. 351-372. [GS SEARCH]

Santos, J., Paiva, R., Bittencout, I. I. (2016). Avaliação Léxico-Sintática de Atividades Escritas em Algoritmo Genético e Processamento de Linguagem Natural: Um Experimento no ENEM. In: Brazilian Journal of Computers in Education, v.24, n.2. doi: 10.5753/rbie.2016.24.02.92. Disponível em: $\underline{\text { http://www.br- }}$ ie.org/pub/index.php/rbie/article/view/6450. [GS SEARCH]

Silva, W. D. C. M., Finger, M. (2013) Improving CoGrOO: the Brazilian Portuguese Grammar Checker. In: Proceedings of the 9th Brazilian Symposium in Information and Human Language Technology, p. 21-29. [GS SEARCH]

Scheuer, O., McLaren, B. M. (2008). Helping Teachers Handle the Flood of Data in Online Student Discussions. In: Intelligent Tutoring Systems, p. 323-332. doi: 10.1007/978-3-54069132-7 36. [GS SEARCH]

Vapnik, V. N. (1995). The nature of Statistical learning theory. Springer-verlag, New York. [GS SEARCH]

Vieira, R., Lima, V. L. S. (2001). Linguística computacional: princípios e aplicações. In: IX Escola de Informática da SBC-Sul. Luciana Nedel (Ed.) Passo Fundo, Maringá, São José. SBC-Sul. [GS SEARCH]

Xia, J. C., Fielder, J., Siragusa, L. (2013). Achieving better peer interaction in online discussion forums: A reflective practitioner case study. In: Issues in Educational Research, v.23, n.1, p. 97-113. Disponível em: https://espace.curtin.edu.au/handle/20.500.11937/13284. [GS $\underline{\mathrm{SEARCH}]}$

Zhang, E., Zhang, Y. (2009). F-measure. In: Encyclopedia of DataBase Systems. Springer US, p. 1147-1147. [GS SEARCH] 